\title{
CDISC SDTM Observational Study Time Perspective Terminology
}

National Cancer Institute

\section{Source}

National Cancer Institute. CDISC SDTM Observational Study Time Perspective

Terminology. NCI Thesaurus. Code C127261.

Terminology associated with the observation study time perspective codelist of the Clinical Data Interchange Standards Consortium (CDISC) Study Data Tabulation Model (SDTM). 